\title{
Atrial fibrillation with pregnancy: A prospective controlled study
}

\author{
Original Yasser Khamis ${ }^{1}$, M. Mabrouk Tohamy ${ }^{2}$ \\ Article \\ ${ }^{1}$ Department of Obstetrics and Gynecology, ${ }^{2}$ Department of Cardiology, Faculty of Medicine, \\ Beni-Suef University, Egypt
}

\begin{abstract}
Background: Atrial fibrillation (AF) has been associated with numerous morbidities and mortalities. Pregnant women are at higher risk to develop AF.

Objective: This study aims at investigating the maternal and fetal complications in women who developed AF during pregnancy compared to a control group.

Patients and Methods: A prospective study was conducted on 2 groups; women with AF and women without AF. The included women were recruited from Beni-Suef University Hospital and Beni-Suef General Hospital after obtaining their informed consents. The maternal and fetal complications have been reported and compared between both groups.

Results: The rates of placental complications, vaginal hematoma, congenital anomalies and the need for neonatal ICU were relatively higher amongst women with AF than those without AF but no statistically significant differences have been noticed $(p>0.05)$. Rupture uterus, abortion and post-partum hemorrhage were statistically higher amongst women with $\mathrm{AF}$ in comparison to women without AF; $37.5 \%$ versus $2.6 \%, 50 \%$ versus $17.4 \%$, and $37.5 \%$ versus $5.3 \%$, respectively $(p<0.05)$. Warfarin, Metoprolol and Digoxin were the most commonly reported medications amongst women with AF. The prescribed medications did not affect the likelihood of maternal or fetal complications $(p>0.05)$.

Conclusion: Women who develop AF during pregnancy are more likely to develop maternal complications.
\end{abstract}

Key Words: Abortion, atrial fibrillation, maternal complications, pregnancy

Received: 24 December 2018, Accepted: 18 January 2019

Corresponding Author:Yasser Khamis, MSc., Department of Obstetrics and Gynecology, Beni-Suef University, Egypt, E-mail: yasserkhicsi1010.yk@gmail.com

ISSN: 2090-7265, May 2019, Vol.9, No. 2

\section{INTRODUCTION}

$\mathrm{AF}$ is a supraventricular arrhythmia that negatively affects the cardiac functions and poses many serious health consequences. It is considered one of the most common and serious arrhythmias that are linked to significant rates of morbidities and mortalities..$^{[1,2]}$

On the other hand, pregnancy is associated with considerable vascular and cardiac changes such as the increase in cardiac output, plasma volume, heart wall tension, heart size and heart rate in addition to the decrease in vascular resistance ${ }^{[3,4]}$. Such changes could induce arrhythmias whether in women with and without a history of arrhythmias and cardiac diseases. ${ }^{[5]}$

AF constitutes a big threat to both mother and fetus. Regarding the pregnant mother, AF increases the risk of many cardiovascular events such as heart failure and stroke and obstetric complications such as premature rupture of membrane (PROM), pregnancyinduced hypertension, placental complications, ruptured uterus, post-partum hemorrhage and vaginal hematoma. Abortion, neonatal death, and the need for neonatal intensive care are of the reported complications of cardiac arrhythmias with pregnancy ${ }^{[6]}$.

Moreover, some of AF medications may carry a teratogenic effect such as Beta-Blockers and Warfarin and the treating physicians always face difficulty in safeguarding the life of the pregnant mothers without exposing the fetuses to the risk of teratogenicity ${ }^{[7,8]}$.

\section{AIM OF THE WORK}

In this regard, this study aimed to follow-up women who developed AF during pregnancy and compare them to women without AF regarding their complications and pregnancy outcome.

\section{PATIENTS AND METHODS}

In this prospective, non-interventional study a total of 20 pregnant women diagnosed with $\mathrm{AF}$ and 50 
pregnant women without $\mathrm{AF}$ who served as controls were followed-up throughout their pregnancy until delivery.

The included women were recruited from Beni-Suef University Hospital and Beni-Suef General Hospital during the period between February and August 2017 and were followed-up for the period of their pregnancy.

Women with other chronic or cardiac diseases rather than AF were excluded from the study. Also, women with twins were not included. Diagnosis of AF was made whether in the first or the second trimester of pregnancy in those with no history of AF before pregnancy. Women who developed AF by the third trimester were not included as well.

The sample size was calculated using Epi-Info version 7 Stat Calc, [Center for Disease Control (CDC), $\mathrm{WHO}$, based on the following criteria; abortion rate of $50 \%$, a confidence level of $95 \%$, a margin of error of $5 \%$ and a loss-to-follow-up of $25 \%$.

Patients were diagnosed with AF using ECG, 24-hour ECG Holter monitor, and echocardiogram by certified emergency specialists and cardiologists from Beni-Suef University Hospital and Beni-Suef General Hospital. A case report form for every woman including her name, date of birth, medical history if present, concomitant disorders, and previous labs if present was done. History of medications and interventions for $\mathrm{AF}$ was also included in the forms.

The following complications were checked and reported; pre-eclampsia, hemolysis elevated liver enzymes low platelets (HELLP) syndrome, pregnancyinduced hypertension, PROM, placental complications, ruptured uterus, and vaginal hematoma.

The protocol of the study has been approved by the Research Ethics Committee of the Faculty of Medicine, Beni-Suef University. The women were informed of the purpose of the study with confirming confidentiality of data and they signed their informed consents before participation.

Data were analyzed using the software, Statistical Package for Social Science (SPSS Inc. Released 2009, PASW Statistics for Windows, version 18.0: SPSS Inc., Chicago, Illinois, USA). Chi-square, odds ratio (OR) and $95 \%$ confidence interval (CI) were calculated whenever needed. P values of less than 0.05 were considered significant.

\section{RESULTS}

Of the 20 women with $\mathrm{AF}$ who were included in the study, $4(20 \%)$ lost-to-follow-up and of the 50 women without AF, 4 (8\%) did not continue the study and left without giving reasons. The mean age of women with AF was $29.7 \pm 6.3$ years; slightly older than those without AF $26.8 \pm 5.1$ years with no statistically significant differences between both groups ( $p>0.05)$. The average of gravidity and parity was lower in women with $\mathrm{AF} 1.5 \pm 0.5$ and $0.9 \pm 0.7$ than women without AF $2.2 \pm 1.6$ and $1.5 \pm 1.2$, however, these differences were statistically insignificant $(p>0.05)$ (Table 1).

Of the 16 women with AF, only $8 \quad(50 \%)$ continued their delivery while $8(50 \%)$ had abortion. Out of the 8 AF patients who continued their pregnancy, 1 (12.5\%) woman had PROM, 1 (12.5\%) placental complication, $3 \quad(37.5 \%)$ ruptured uterus, $2(25 \%)$ vaginal hematoma, $1(12.5 \%)$ congenital anomalies. After giving birth, 3 (37.5\%) women had post-partum bleeding (Table 2, Figures 1-3).

On the other hand, out of the 46 women without AF, $38(82.6 \%)$ continued their pregnancy and $8(17.4 \%)$ had abortion. Of those 38 women, $2(5.3 \%)$ had PROM, 2 (5.3\%) placental complications, 1 (2.6\%) ruptured uterus, 1 (2.6\%) vaginal hematoma, $3(7.9 \%)$ congenital anomalies, and $2(5.3 \%)$ post-partum bleeding (Table 2, Figures 1-3).

Preeclampsia, HELLP and pregnancy-induced hypertension were not reported in both groups. The need for neonatal ICU was recorded in $1(12.5 \%)$ woman with AF and $2(5.3 \%)$ women without AF (Table 2).

Although most complications were more obvious in women with AF, only rupture uterus, abortion and post-partum bleeding showed statistically significant differences $(p<0.05)$ (Table 2, Figures 1-3).

Half of the women with AF was diagnosed during their first trimester while the other half was diagnosed during the second trimester. No considerable differences regarding the obstetric history, complications, and pregnancy outcome between women who developed their AF during the first trimester and those who were diagnosed with AF during the second trimester $(p>0.05)$

The results also showed that 12 women with AF were prescribed for Warfarin, 7 Metoprolol, 6 Digoxin, 6 Diltiazem, 3 Verapamil, and 1 Procainamide (Figure 4). 
Table 1: Comparison between women with and without AF regarding their age and obstetric code

\begin{tabular}{lccc}
\hline Characters & AF & No AF & P value \\
\hline Age $($ Mean \pm Sd) & $29.7 \pm 6.3$ & $26.8 \pm 5.1$ & 0.074 \\
Gravidity $($ Mean \pm Sd) & $1.5 \pm 0.5$ & $2.2 \pm 1.6$ & 0.126 \\
Parity $($ Mean \pm Sd) & $0.9 \pm 0.7$ & $1.5 \pm 1.2$ & 0.068 \\
Abortion $($ Mean \pm Sd) & $0.6 \pm 0.6$ & $0.7 \pm 1.0$ & 0.093 \\
\hline
\end{tabular}

Table 2: Comparison between women with and without AF regarding their maternal and fetal complications

\begin{tabular}{|c|c|c|c|c|}
\hline Complications & $\mathrm{AF}, \mathrm{n}=8(\%)$ & No $A F, n=38(\%)$ & $\mathrm{P}$ value & OR $(95 \% \mathrm{CI})$ \\
\hline Pre-eclampsia & $0(0.0)$ & $0(0.0)$ & --- & --- \\
\hline Hypertension & $0(0.0)$ & $0(0.0)$ & --- & --- \\
\hline HELLP & $0(0.0)$ & $0(0.0)$ & --- & --- \\
\hline PROM & $1(12.5)$ & $2(5.3)$ & 0.444 & $2.0(0.4-11.6)$ \\
\hline Placental complications & $1(12.5)$ & $2(5.3)$ & 0.444 & $1.3(0.6-2.8)$ \\
\hline Ruptured uterus & $3(37.5)$ & $1(2.6)$ & $0.013 *$ & $6.3(2.3-17.1)$ \\
\hline Vaginal hematoma & $2(25.0)$ & $1(2.6)$ & 0.074 & $4.8(1.6-14.2)$ \\
\hline Congenital anomalies & $1(12.5)$ & $3(7.9)$ & 0.548 & $1.1(0.6-2.0)$ \\
\hline Need for neonatal ICU & $1(12.5)$ & $2(5.3)$ & 0.444 & $1.3(0.6-2.8)$ \\
\hline
\end{tabular}

*P value is considered significant

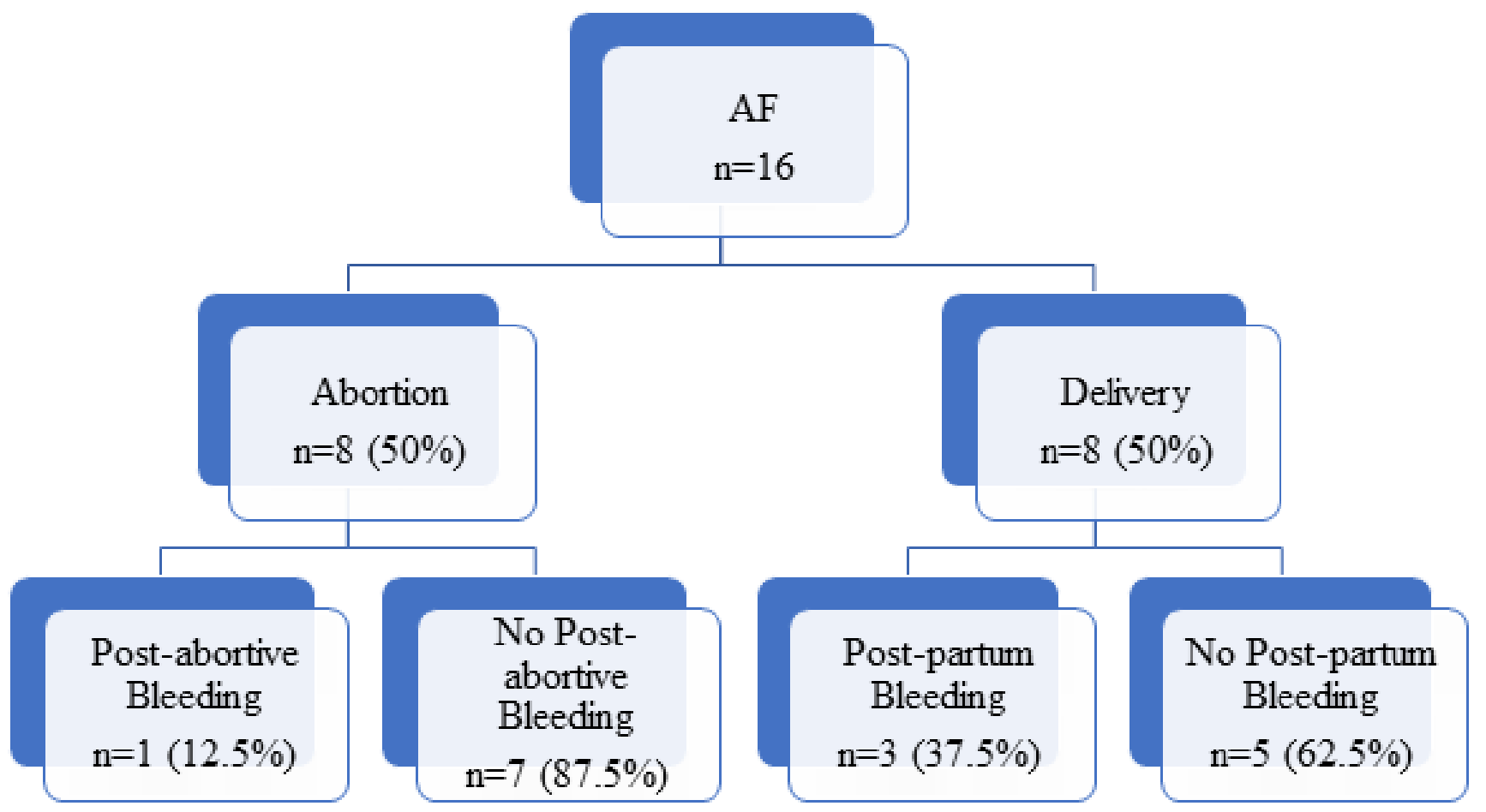

Fig. 1: Outcome of pregnant women with AF 


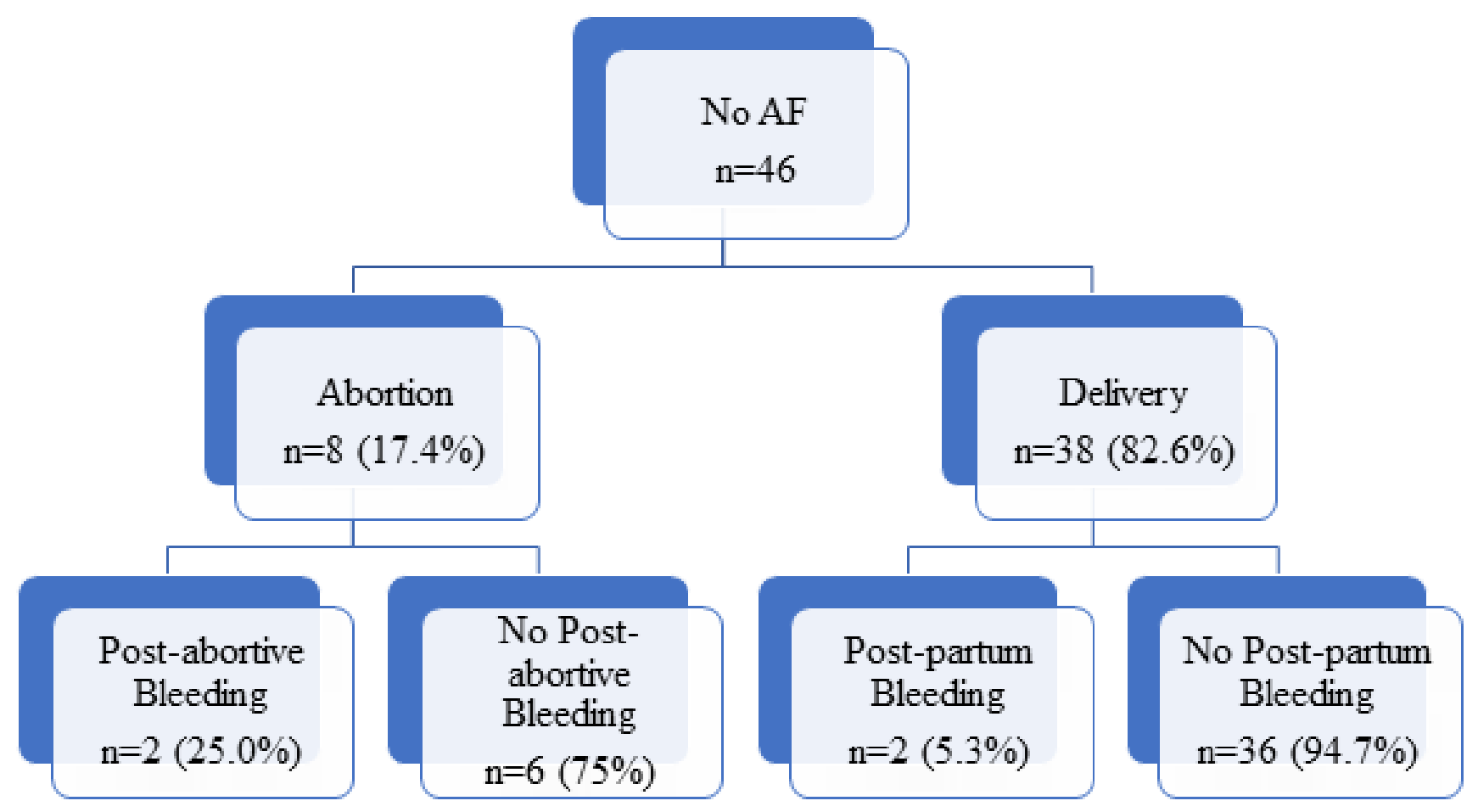

Fig. 2: Outcome of pregnant women without AF

$$
\text { 틀 } \mathrm{AF} \text { № AF }
$$

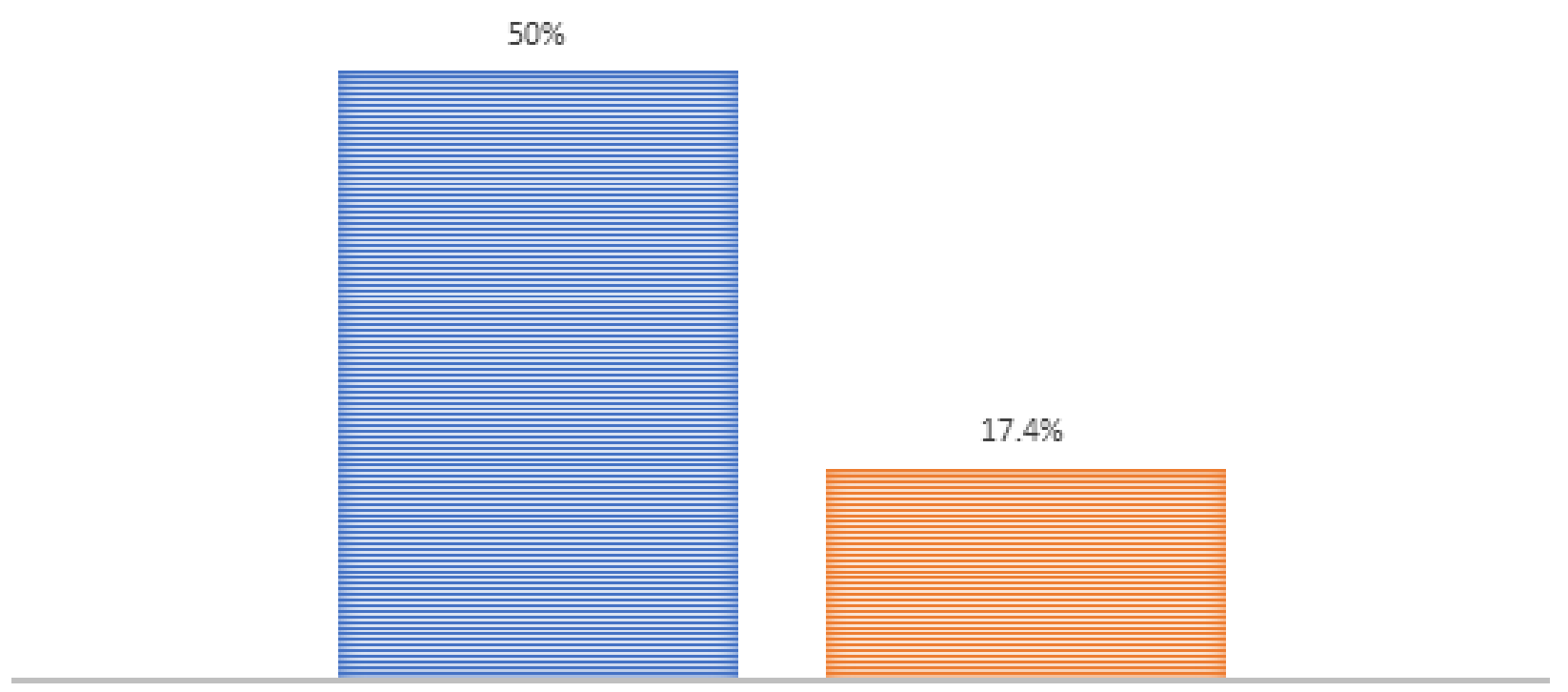

Abortion Rate

Fig. 3: Comparison between women with and without $\mathrm{AF}$ regarding the fetal outcome 


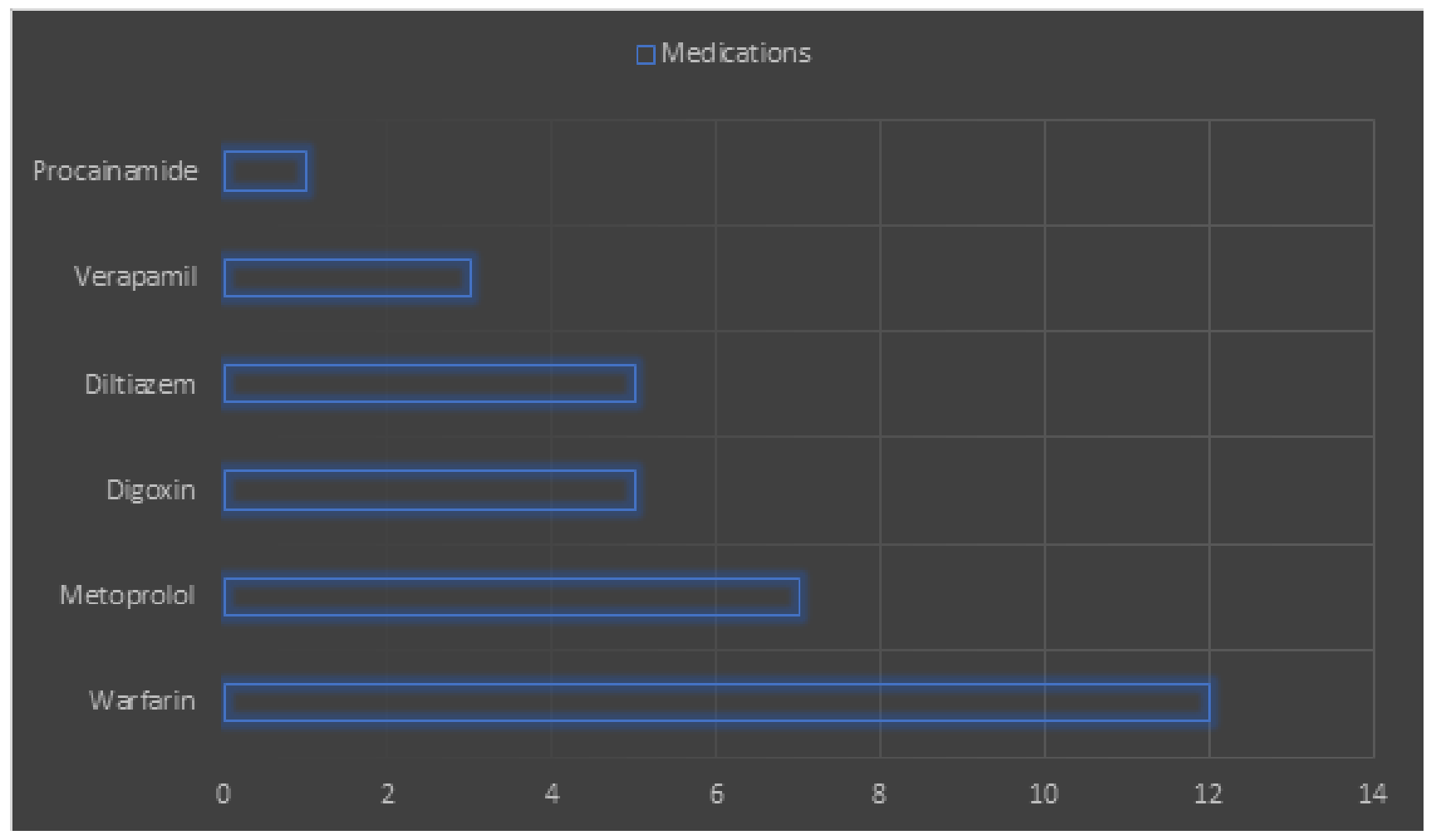

Fig. 4: Medications prescribed to women with AF (women can be prescribed to more than 1 medication)

\section{DISCUSSION}

This study investigated the association between developing AF during pregnancy and maternal and fetal complications using a longitudinal design. The association between cardiac arrhythmias and pregnancy has been attributed to the cardiovascular changes that develop during pregnancy such as the increased cardiac size, heart rate, and cardiac output compared to the reduction in systemic resistance could induce atrial stretches and arrhythmia ${ }^{[5]}$.

Generally, AF could lead to serious cardiac events by 2 main ways; the rising heart rate without effective atrial contraction may lead to heart failure, in addition to the synergistic effect of the ineffective atrial contraction and the physiological prothrombotic status in pregnancy that could increase the risk of thromboembolism ${ }^{[9]}$. However, no serious cardiac events have been reported in our sample. Previous studies that reported cardiac complications from AF during pregnancy showed very low rates of complications and most of these complications were primarily attributed to structural disorders that aggravated the arrhythmia during pregnancy ${ }^{[6,10]}$.

Interestingly, another study on women with a history of tachyarrhythmia showed that those women were more likely to develop arrhythmias during pregnancy ${ }^{[11]}$.
In this study, though statistically insignificant, women with AF were considerably older than those without AF in our study. Previous studies linking the age of the pregnant women and $\mathrm{AF}$ showed that women with older age were more likely to develop $\mathrm{AF}^{[2,6]}$. However, it should be noticed that the old age is a well-known risk factor for AF regardless of the pregnancy status ${ }^{[12]}$.

Our study showed that AF could be associated with many maternal complications such as rupture membrane, PROM, post-partum hemorrhage, placental complications and abortion. A previous community-based study could name PROM, placental abruption, and post-partum hemorrhage as obstetric complications in women with AF; however, the study reported pregnancy-induced hypertension in more than $10 \%$ of women with $\mathrm{AF}$ compared to no hypertension in our study ${ }^{[6]}$. Another study on women with preexisting arrhythmias with exacerbation during pregnancy showed that the recurrence of arrhythmia could be an independent risk factor for maternal and fetal complications $^{[11]}$.

Women with FA in our study admitted taking anti-coagulant and anti-arrhythmic medications which coincided with previous reports ${ }^{[6]}$. Our findings agree with the current guidelines for AF with pregnancy ${ }^{[2]}$. However, it is worth pointing out that management of cardiac arrhythmias during pregnancy is not typically similar to that of patients with no pregnancy. The ultimate goal of such medications is to control the heart rate, restore the 
sinus rhythm, and minimize the hypercoagulation status in order to protect the pregnant woman and the fetus ${ }^{[4]}$.

\section{CONCLUSION AND RECOMMENDATION}

In conclusion, AF during pregnancy is associated with serious maternal complications and could affect the fetus. Further interest should be given to screening of pregnant women for cardiac arrhythmias.

\section{CONFLICT OF INTEREST}

There are no conflict of interests.

\section{REFERENCES}

1. Gutierrez C, Blanchard DG. Diagnosis and Treatment of Atrial Fibrillation. Am Fam Physician. 2016;94(6):442-52.

2. January CT, Wann LS, Alpert JS, Calkins H, Cigarroa JE, Cleveland JC Jr, et al. American College of Cardiology/American Heart Association Task Force on Practice G. 2014 AHA/ACC/HRS guideline for the management of patients with atrial fibrillation: a report of the American College of Cardiology/American Heart Association Task Force on Practice Guidelines and the Heart Rhythm Society. J Am Coll Cardiol. 2014; 64:1-76.

3. Ouzounian JG, Elkayam U. Physiologic changes during normal pregnancy and delivery. Cardiol Clin. 2012;30: 317-29.

4. Gilson GJ, Samaan S, Crawford MH, Qualls CR, Curet LB. Changes in hemodynamics, ventricular remodeling, and ventricular contractility during normal pregnancy: a longitudinal study. Obstet Gynecol. 1997; 89:957-62.
5. Knotts RJ, Garan H. Cardiac arrhythmias in pregnancy. Semin Perinatol. 2014; 38:285-8.

6. Lee M, Chen W, Zhang Z, Duan L, Ng A, Spencer $\mathrm{H}$, et al. Atrial fibrillation and atrial flutter in pregnant women- a population-based study. Am Heart Assoc. 2016;5: e003182.

7. Vitale N, De Feo M, De Santo LS, Pollice A, Tedesco N, Cotrufo M. Dose dependent fetal complications of warfarin in pregnant women with mechanical heart valves. J Am Coll Cardiol. 1999;33: 1637-41.

8. Lydakis C, Lip GY, Beevers M, Beevers DG. Atenolol and fetal growth in pregnancies complicated by hypertension. Am J Hypertens. 1999; 12:541-7.

9. Cordina R, McGuire M. Maternal cardiac arrhythmias during pregnancy and lactation. Obstet Med. 2010; 3(1): 8-16.

10. Salam AM, Ertekin E, van Hagen IM, Suwaidi JA, Ruys TPE, Johnson MR, et al. Atrial fibrillation of flutter during pregnancy in patients with structural heart disease. JACC Clin Electrophysiol. 2015;1: 284-92.

11. Silversides CK, Harris L, Haberer K, Sermer M, Colman JM, Siu SC. Recurrence rates of arrhythmias during pregnancy in women with previous tachyarrhythmia and impact on fetal and neonatal outcomes. Am J Cardiol. 2006;97: 1206-12.

12. Go AS, Hylek EM, Phillips KA, Chang Y, Henault LE, Selby JV, Singer DE. Prevalence of diagnosed atrial fibrillation in adults: national implications for rhythm management and stroke prevention: the anticoagulation and risk factors in atrial fibrillation (ATRIA) study. JAMA. 2001; 285:2370-5. 